\title{
MINERALIZAÇÃO E SORÇÃO DO FUNGICIDA FLUOPICOLIDA DE AMOSTRAS DE SOLOS NEOSSOLO E LATOSSOLO EM ÁREAS PRESERVADAS E ÁREAS TRATADAS
}

\author{
LUCILENE YURIKO MURAKAMI* \\ REGINA C. B. FERREIRA** \\ GISELE S. SOUZA*** \\ LUIZ C. LUCHINI ${ }^{* * * *}$
}

\begin{abstract}
O objetivo deste estudo foi verificar a mineralização e a sorção do fungicida fluopicolida em Neossolo e Latossolo de áreas preservadas e áreas de uso agrícola, visando comparar e identificar se há alteração no comportamento do agrotóxico em diferentes solos e áreas. Para todas as amostras de solo, as taxas de mineralização foram $<20 \%$, sendo que para o Neossolo houve melhor mineralização na área preservada em relação a sua respectiva área agrícola. Esse comportamento não foi observado para o Latossolo, pois as duas áreas apresentaram comportamento de mineralização similar. Os resultados do coeficiente de adsorção demonstraram média e baixa adsorção para Latossolo e Neossolo, respectivamente, independente da área coletada e as isotermas de sorção do composto revelaram-se bem ajustadas ao modelo de Freundlich, com valores de $1 / \mathrm{n}$ em torno de 0,9 . Baseado nesses resultados pode-se considerar o fluopicolida como fungicida de mineralização lenta e de média mobilidade em Neossolo e Latossolo em áreas preservadas e agrícolas. Concluiu-se que o fluopicolida não exerce efeito adverso em áreas nas quais há constante manejo agrícola em razão de seu comportamento similar em área preservada.
\end{abstract}

PALAVRAS-CHAVE: FUNGICIDA; MINERALIZAÇÃO; SORÇÃO; SOLOS; FLUOPICOLIDA.

* Pós-Graduanda em Sanidade, Segurança Alimentar e Ambiental no Agronegócio, Laboratório de Ecologia de Agroquímicos, Instituto Biológico (IB), São Paulo, SP (e-mail: luciym@gmail.com).

** Química, Laboratório de Ecologia de Agroquímicos, IB, São Paulo, SP (e-mail: reginacbf@jg.com.br).

*** Licenciada em Química, Laboratório de Ecologia de Agroquímicos, IB, São Paulo, SP (e-mail: gisa_ vedder@hotmail.com).

**** Doutor em Química Analítica, Laboratório de Ecologia de Agroquímicos, IB, São Paulo, SP (e-mail: luchini@biologico.sp.gov.br). 


\section{INTRODUÇÃo}

O crescimento da população mundial tem forçado o aumento da produção de alimentos mediante a abertura de novas fronteiras agrícolas e da intensificação do uso do solo, adotandose tecnologias como, a utilização de fertilizantes, variedades melhoradas, plantios adensados, agroquímicos e a introdução de plantas transgênicas, dentre outras (OLIVEIRA e BRIGHENT, 2011). Assim, houve grande aumento no consumo de agrotóxicos para a produção em ampla escala de determinadas culturas vegetais e a criação de espécies de animais de interesse econômico. Elevado consumo de agrotóxicos aumenta a possibilidade de ocorrência de contaminação ambiental, de animais e do homem.

Depois da aplicação de agrotóxicos, vários processos físicos, químicos, físico-químicos e biológicos determinam seu comportamento. Além disso, o destino de agrotóxicos no ambiente é governado por processos de sua retenção nas partículas do solo (sorção, adsorção), de transformação (degradação química e biológica) e de transporte (deriva, volatilização, lixiviação e carreamento superficial), e ainda por interações desses processos (SPADOTTO et al., 2004).

As modificações que podem ocorrer com as moléculas dos agrotóxicos no solo podem envolver: a quebra total da molécula (chamada de mineralização), dando origem a $\mathrm{CO}_{2}, \mathrm{H}_{2} \mathrm{O}$ e íons minerais; ou parcial, dando origem a subprodutos (chamada de metabolização). Tais modificações ocorrem por meios bióticos ou abióticos (COX et al., 1997), por isso estudos do comportamento desses compostos no ambiente têm como principais objetivos: descobrir os fatores que influenciam direta ou indiretamente a eficácia de ação do produto sobre a praga, assim como as possíveis interações dos agrotóxicos com o meio de forma a minimizar os efeitos adversos causados pela presença desses produtos no solo (LAVORENTI, 1996).

Segundo Luchini e Andréa (2002), a adsorção dos agrotóxicos no solo normalmente constitui processo chave no comportamento desses compostos, pois está diretamente relacionada com a translocação, a persistência, a mobilidade e a bioatividade dos compostos nos solos e plantas ali presentes. A adsorção de agrotóxicos no solo assume importância principalmente por se relacionar diretamente com os processos de disponibilidade para a atividade do composto, para o ataque microbiano e biodegradação, e inversamente com a possibilidade de lixiviação no perfil do solo e potencial de poluição de águas superficiais e subterrâneas. Além disso, sua ocorrência pode variar enormemente em função das diferentes propriedades físicas e químicas dos solos, como conteúdo orgânico, pH, quantidade e tipos de argilas, etc. O processo de adsorção desses produtos no solo determina a fração do agrotóxico disponível para sua ação biológica, já que o processo de adsorção resulta da sua partição entre a fase sólida e a fase líquida do solo. A adsorção de agrotóxicos pode ser representada pela equação de Freundlich para o equilíbrio produto-superfície do solo-solução do solo: $\log x / m=\log K f+1 / n \log C e$, em que $x / m$ corresponde à concentração de equilíbrio na fase sólida do solo, Ce representa a concentração de equilíbrio em solução, e Kf e 1/n são constantes empíricas (GREEN e KARICKHOFF, 1990).

O fungicida fluopicolida tem sido utilizado no Brasil nas culturas de batata, fumo, melão, tomate e uva, conforme o sistema de consulta pública Agrofit do Ministério de Agricultura, Pecuária e Abastecimento (MAPA). Atua de modo sistêmico via xilema e translaminar. As propriedades físico-químicas do fluopicolida (como log $\mathrm{K}_{\text {ow }}=3,26$ e $\left.\mathrm{DT}_{50 \mathrm{lab} 20^{\circ} \mathrm{C}} \mathrm{(t} \frac{1}{2}\right)$ no solo em condições de laboratório de 194 dias - 411 dias) indicam sorção média do composto nos coloides do solo e baixa degradação. Dessa forma, é necessário o desenvolvimento de trabalhos para verificar o comportamento desse fungicida em solos brasileiros.

Os objetivos deste trabalho foram verificar a mineralização e a adsorção do fungicida fluopicolida nos solos Neossolo e Latossolo em áreas preservadas e áreas agrícolas. 


\section{MATERIAL E MÉTODOS}

\subsection{FUNGICIDA FLUOPICOLIDA}

Foram utilizados o fluopicolida padrão analítico (lote MOY4627, pureza 99,2 \%) e radiomarcado [Pyridyl-2,6- ${ }^{-14} \mathrm{C}$ ] fluopicolida (ID: KML 9513, atividade específica $5.73 \mathrm{MBq}$ ).

\subsection{AMOSTRAS DE SOLO}

Para realização do estudo coletaram-se amostras superficiais de Latossolo e Neossolo $(0-5 \mathrm{~cm})$ de áreas "off crop" (preservadas) e áreas "in crop" (tratadas), localizadas no munícipio de Piracicaba (SP), cujas coordenadas geográficas estão na Tabela 1.

\section{TABELA 1 - COORDENADAS GEOGRÁFICAS DAS REGIÕES DOS SOLOS ESTUDADOS}

\begin{tabular}{|c|c|c|}
\hline & "off crop" & "in crop" \\
\hline \multirow{2}{*}{ Latossolo } & $22^{\circ} 37^{\prime} 19^{\prime \prime} \mathrm{S}$ & $22^{\circ} 43^{\prime} 1.4^{\prime \prime} \mathrm{S}$ \\
\hline & $47^{\circ} 36^{\prime} 45^{\prime \prime} \mathrm{W}$ & $53^{\circ} 53^{\prime} 43.2^{\prime \prime} \mathrm{W}$ \\
\hline \multirow{2}{*}{ Neossolo } & $22^{\circ} 43^{\prime} 32^{\prime \prime} \mathrm{S}$ & $22^{\circ} 43^{\prime} 1.4^{\prime \prime} \mathrm{S}$ \\
\hline & $48^{\circ} 1^{\prime} 21^{\prime \prime} \mathrm{W}$ & $48^{\circ} 1$ ' $10.55^{\prime \prime} \mathrm{W}$ \\
\hline
\end{tabular}

Os solos foram escolhidos por serem tipicamente tropicais e coletados à profundidade de $0-5 \mathrm{~cm}$, na qual ocorre maior atividade microbiana e há maior conteúdo de matéria orgânica, pois esses parâmetros edáficos são de fundamental importância nos processos de degradação e de adsorção dos agrotóxicos.

As propriedades físicas e químicas, assim como a análise química para avaliação de fertilidade dos solos (pois nas áreas "in crop" é comum a prática de fertilização), foram realizadas nos laboratórios do Departamento de Ciência de Solo, da Escola Superior de Agricultura Luiz de Queiroz, da Universidade de São Paulo (ESALQ/USP) e seus parâmetros estão apresentados nas Tabelas 2 e 3.

TABELA 2 - ANÁLISE QUÍMICA DOS SOLOS

\begin{tabular}{ccccc}
\hline & $\mathrm{pH} \mathrm{H}_{2} \mathrm{O}$ & $\mathrm{pH} \mathrm{KCl}$ & $\begin{array}{c}\mathrm{MO} \\
\mathbf{g ~ d m}^{-3}\end{array}$ & $\begin{array}{c}\mathbf{N} \\
\text { (total) } \\
\mathbf{~ m g ~ k g ~}^{-1}\end{array}$ \\
\hline Latossolo "off crop" & 5,6 & 4,5 & 54,0 & $3.682,0$ \\
\hline Latossolo "in crop" & 5,7 & 4,6 & 34,0 & $1.505,0$ \\
\hline Neossolo "off crop" & 5,4 & 4,3 & 24,0 & $1.120,0$ \\
\hline Neossolo "in crop" & 6,4 & 6,2 & 16,0 & 602,0 \\
\hline
\end{tabular}

$\mathrm{MO}=$ Matéria orgânica .

Após a coleta, as amostras de solos foram espalhadas em superfície plana e secaram ao ar ambiente durante três dias. Os solos secos foram peneirados em malha de $2 \mathrm{~mm}$ e quando 
se apresentavam "empedrados" submetidos ao destorroamento, utilizando-se pistilo e cadinho. As amostras foram armazenadas em geladeira à temperatura de aproximadamente $4{ }^{\circ} \mathrm{C}$, com umidade natural por período não superior a 4 meses. Parte dessas amostras foi armazenada por duas semanas em câmara de incubação (BOD) à aproximadamente $25^{\circ} \mathrm{C}$ com água suficiente para umedecê-las e reativá-las.

TABELA 3 - RESULTADOS DA ANÁLISE FÍSICA DOS SOLOS

\begin{tabular}{lcccc}
\hline & Areia Total & $\begin{array}{c}\text { Silte } \\
\left(\mathbf{g ~ k g}^{-1}\right)\end{array}$ & $\begin{array}{c}\text { Argilas } \\
\text { c/disp. }\end{array}$ & $\begin{array}{c}\text { Classe de } \\
\text { textura }\end{array}$ \\
\hline $\begin{array}{l}\text { Latossolo "off } \\
\text { crop" }\end{array}$ & 261,0 & 92,0 & 647 & m-arg. \\
\hline $\begin{array}{l}\text { Latossolo "in } \\
\text { crop" }\end{array}$ & 378,0 & 53,0 & 569 & arg. \\
\hline $\begin{array}{l}\text { Neossolo "off } \\
\text { crop" }\end{array}$ & 877,0 & 23,0 & 100 & ar. \\
\hline $\begin{array}{l}\text { Neossolo "in } \\
\text { crop" }\end{array}$ & 924,0 & 13,0 & 63 & ar. \\
\hline
\end{tabular}

\subsection{CAPACIDADE MÁXIMA DE RETENÇÃO DE ÁGUA (CMRA)}

Para a determinação da capacidade máxima de retenção de água (CMRA) adaptou-se a metodologia da EMBRAPA (FRIGHETTO e VALARINI, 2000). Pesaram-se três replicatas de $10,0 \mathrm{~g}$ de cada solo peneirado e seco, que foram transferidas para três funis analíticos apoiados em provetas cuja saída estava vedada com pequena quantidade de lã de vidro. Adicionaram-se 10,0 mL de água em cada replicata de solo e aguardou-se a água toda escoar. Anotou-se o volume de água obtido na proveta e efetuou-se seu descarte. Repetiu-se o procedimento de adição de água até o momento que o volume de $10,0 \mathrm{~mL}$ fosse percolado.

Somou-se todo o volume de água retido de cada replicata e efetuou-se a média aritmética entre elas. O valor obtido representa a capacidade máxima de retenção de água ( $100 \%$ da CMRA) para $10,0 \mathrm{~g}$ de solo. Dividiu-se o valor obtido por 10 para expressar o valor por grama de solo.

\subsection{CONTEÚDO DE ÁGUA PRESENTE NO SOLO}

Pesaram-se três replicatas de 3,0 $\mathrm{g}$ de cada solo úmido em analisador de umidade (Metler, LJ16). Cada replicata permaneceu no aparelho durante 20 minutos a $120^{\circ} \mathrm{C}$. Os valores encontrados foram utilizados para corrigir o conteúdo de água no solo e ajustar a porcentagem de CMRA a ser utilizada no estudo.

\subsection{AJUSTE DA QUANTIDADE DE ÁGUA NO SOLO A 60 \% CMRA}

O valor da umidade por grama de solo calculado no item 2.3 equivale a $100 \%$ da quantidade de água retida no solo. Calculou-se o valor equivalente a $60 \%$ por meio de regra de três simples e descontou-se o valor obtido no item 2.4, cujo resultado equivale à quantidade de água adicionada por grama de solo para que se obtivesse $60 \%$ de CMRA.

\subsection{MINERALIZAÇÃO DE FLUOPICOLIDA}

Para o estudo de mineralização do fluopicolida, $50 \mathrm{~g}$ (base seca) de solo úmido foram acondicionados em frascos biométricos, sendo três replicatas para cada período de incubação 
$(0,30,60$ e 130 dias). Transferiu-se para cada frasco, $500 \mu \mathrm{L}$ da mistura de solução de fluopicolida (radiomarcado e não marcado) preparada em metanol/ $\mathrm{H}_{2} \mathrm{O}(1: 1, \mathrm{v} / \mathrm{v})$, com atividade de $0,1185 \mathrm{MBq} \mathrm{mL}^{-1}$ do produto radioativo e concentração total de $20 \mu \mathrm{g} \mathrm{mL}^{-1}$, equivalente à dose final de $120 \mathrm{~g}_{\text {ia ha-1}}$ (dose máxima de campo recomendada por aplicação). Aplicou-se a solução diretamente sobre a superfície das amostras de solos, previamente úmidos. Após homogeneização, as amostras foram ajustadas à umidade do solo para $60 \%$ da CMRA.

$\mathrm{Na}$ lateral do frasco biométrico adicionaram-se $10 \mathrm{~mL}$ de solução $\mathrm{KOH} 0,1 \mathrm{M}$ para a captura do ${ }^{14} \mathrm{CO}_{2}$, proveniente da mineralização do fluopicolida radiomarcado. Trocou-se essa solução diariamente até o oitavo dia e semanalmente no intervalo de 15, 22, 28, 36, 43, 50, 60, 64, 71, $78,85,92,99,106,113$ e 130 dias após o tratamento e início da incubação para a determinação do ${ }^{14} \mathrm{CO}_{2}$ formado. Nesse procedimento houve a troca de toda a solução de $\mathrm{KOH} 0,1 \mathrm{M}$ que estava no frasco mediante adição de solução fresca. Da solução retirada, transferiram-se três alíquotas de 2,0 $\mathrm{mL}$ para frascos contendo $10 \mathrm{~mL}$ de solução líquida cintiladora para amostras aquosas de Mesquita e Rüegg (1984). As amostras foram agitadas, mantidas no escuro por no mínimo 24 horas para leitura em Contador de Cintilação Líquida (CCL), equipamento Packard (1600 TR), durante 10 minutos. Essa técnica baseia-se na reação do $\mathrm{KOH}$ com o ${ }^{14} \mathrm{CO}_{2}$ resultante da mineralização do fungicida pela microbiota do solo, conforme o protocolo 304A da OECD (1981).

\subsection{EXTRAÇÃO DO ${ }^{14} \mathrm{C}-$ FLUOPICOLIDA NAS AMOSTRAS DE SOLO}

Para a determinação das frações extraídas e não extraídas dos produtos de degradação do fungicida fluopicolida radiomarcado, as amostras de solo foram retiradas dos frascos biométricos após cada intervalo de incubação, correspondente a 0, 28, 60 e 130 dias após a aplicação do fungicida. Extraiu-se o solo com $150 \mathrm{~mL}$ de acetonitrila em Soxhlet a $70^{\circ} \mathrm{C}$ durante 8 horas. $O$ volume desse extrato foi medido e 3 alíquotas de $1,0 \mathrm{~mL}$ cada foram transferidas para frascos contendo $10 \mathrm{~mL}$ de solução líquida cintiladora para amostras orgânicas de Mesquita e Rüegg (1984). Os frascos foram agitados, mantidos no escuro por no mínimo 24 horas e levados para leitura em CCL. O restante dos extratos foi armazenado em geladeira a $4{ }^{\circ} \mathrm{C}$ como amostras sobressalentes.

\subsection{DETERMINAÇÃO DOS RESÍDUOS LIGADOS DE ${ }^{14} \mathrm{C}-$ FLUOPICOLIDA}

Após a extração em Soxhlet com acetonitrila, os solos foram secos ao ar livre por 7 dias (ou secagem completa) e mantidos armazenados em geladeira a $4{ }^{\circ} \mathrm{C}$ até o momento da análise.

Para a análise dos ${ }^{14} \mathrm{C}$-resíduos ligados ainda presentes no solo após a extração com solvente, $500 \mathrm{mg}$ desses solos em triplicata foram oxidados a $900{ }^{\circ} \mathrm{C}$ com fluxo de $380 \mathrm{~cm}^{3} / \mathrm{min}$ de $\mathrm{N}_{2}$ e o mesmo fluxo de $\mathrm{O}_{2}$ por 4 minutos, em oxidador biológico (Biological Oxidizer, OX600). Coletouse o dióxido de carbono radiomarcado, proveniente da queima dos ${ }^{14} \mathrm{C}$-compostos presentes no solo, em armadilha contendo $3,0 \mathrm{~mL}$ de monoetanolamina e $12 \mathrm{~mL}$ de solução cintiladora para solvente aquoso misturada com metanol na proporção 6:4. A radioatividade presente foi detectada por $\mathrm{CCL}$, para o fechamento do balanço de radioatividade como ${ }^{14} \mathrm{CO}_{2}+{ }^{14} \mathrm{C}$-extraível $+{ }^{14} \mathrm{C}$-ligado. (NAKAGAWA \& ANDRÉA, 2000).

As médias das replicatas dos resultados das mineralizações do fluopicolida foram comparadas mediante análise de variância ANOVA pelo programa Excel (MICROSOFT, 2010).

\subsection{ENSAIOS DE ADSORÇÃO E DESSORÇÃO}

Utilizou-se o método "batch equilibrium" para a realização do ensaio de adsorção do ${ }^{14} \mathrm{C}$-fluopicolida em amostras de solos, baseado no protocolo da 106 OECD (2000). Triplicatas de $1,0 \mathrm{~g}$ de solo (base seca) foram colocadas em tubos de centrífuga de polietileno com capacidade de $15 \mathrm{~mL}$, sendo adicionados $10 \mathrm{~mL}$ de solução de fluopicolida nas concentrações de 0,$0066 ; 0,01$; 0,02; 0,04 e 0,08 $\mu$ g i.a mL-1 , preparadas em solução CaCl2 0,01M como eletrólito-suporte, utilizando 
o padrão analítico e ${ }^{14} \mathrm{C}$-fluopicolida $0,053 \mu \mathrm{ci} \mathrm{mL} \mathrm{m}^{-1}$. A concentração de $0,02 \mu \mathrm{g}$ i.a mL $\mathrm{mL}^{-1}$ correspondeu

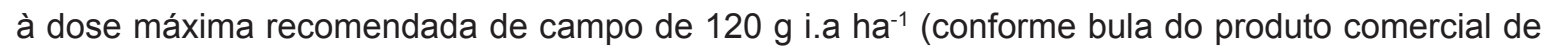
maior dose registrada), levando-se em conta densidade do solo de $1,0 \mathrm{~g} \mathrm{~cm}^{-3}$ e profundidade de $5 \mathrm{~cm}$.

Os frascos de polietileno contendo o sistema solo/solução foram agitados por 24 horas em agitador horizontal. Após esse período, os frascos foram centrifugados a $2000 \mathrm{rpm}$ por 20 minutos e três alíquotas do sobrenadante retiradas e colocadas cada uma em frasco de solução líquida cintiladora para amostras aquosa de Mesquita e Rüegg (1984). As amostras foram agitadas e mantidas no escuro por no mínimo 24 horas para a leitura em CCL (Contador de Cintilação Líquida - Equipamento Packard, 1600 TR), durante 10 minutos.

Calculou-se a quantidade de fluopicolida adsorvida aos solos (Cs) em $\mu_{\text {g g }}{ }^{-1}$ pela diferença entre a quantidade de solução-padrão inicialmente adicionada ao solo (Ci) em $\mathrm{mg} \mathrm{L}^{-1}$ e a quantidade encontrada na solução de equilíbrio $(\mathrm{Ce}) \mathrm{em} \mathrm{mg} \mathrm{L}^{-1}$, conforme a equação 1 :

$$
\mathrm{Cs}=(\mathrm{Ci}-\mathrm{Ce}) \mathrm{v} / \mathrm{m}
$$

Em que:

Cs = Quantidade de fluopicolida sorvida $\left(\mu \mathrm{g} \mathrm{g}{ }^{-1}\right)$;

$\mathrm{Ci}=$ Concentração inicial do fluopicolida $\left(\mathrm{mg} \mathrm{L}^{-1}\right)$;

$\mathrm{Ce}=$ Concentração do fluopicolida em equilíbrio $\left(\mathrm{mg} \mathrm{L}^{-1}\right)$;

$v=$ Volume da solução de fluopicolida (L);

$m=$ massa do solo $(\mathrm{kg})$.

Os resultados da sorção do fluopicolida foram ajustados à equação linearizada de Freundlich de acordo com a equação 2 :

$$
\log C s=\log K_{f}+1 / n \cdot \log C e
$$

Em que:

Cs = Quantidade de fluopicolida sorvida por massa de solo $\left(\mu \mathrm{g} \mathrm{g}^{-1}\right)$;

$K_{f}=$ Constante de Freundlich;

$\mathrm{Ce}=$ Concentração do fluopicolida em equilíbrio $\left(\mathrm{mg} \mathrm{L}^{-1}\right)$;

$1 / n=$ Grau de linearidade da isoterma de sorção.

O coeficiente de sorção $\left(\mathrm{K}_{\mathrm{f}}\right)$ foi normalizado para o teor de CO (carbono orgânico) do solo, obtendo-se o valor de $\mathrm{K}_{\mathrm{foc}}$ pela equação 3 :

$$
\mathrm{K}_{\mathrm{foc}}=\mathrm{K}_{\mathrm{p}} / \mathrm{CO} \times 100
$$

Em que:

Teor de $\mathrm{CO}=\mathrm{MO} / 1,724 ; \mathrm{MO}=$ matéria orgânica.

Sequencialmente à sorção realizou-se o ensaio de dessorção do fluopicolida dos solos para as concentrações utilizadas na adsorção. A solução sobrenadante dos respectivos tubos contendo solo/solução da adsorção foi transferida para outros tubos e armazenada em geladeira. Ao solo remanescente foram adicionadas alíquotas de $10 \mathrm{~mL}$ de $\mathrm{CaCl}_{2} 0,01 \mathrm{M}$. Os tubos que continham o fluopicolida retido às partículas dos solos e a nova solução de cloreto de cálcio foram agitados durante 24 horas. Posteriormente, os frascos foram centrifugados por 20 min a 2000 rpm e alíquotas de 1,0 mL cada, em triplicata, foram analisadas por CCL (mesmo procedimento da sorção).

Calculou-se a quantidade do fluopicolida dessorvida dos solos para a solução aquosa de $\mathrm{CaCl}_{2}$ 0,01M, livre do fungicida, diretamente pela diferença entre a concentração do fungicida no solo antes das etapas de dessorção e a concentração da solução analisada após o equilíbrio de 24 horas, utilizando-se a equação 4 : 


$$
A_{D}=\text { Ced. } V
$$

Em que:

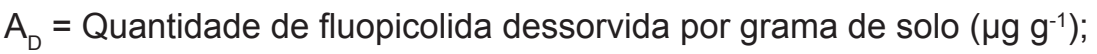

Ced $=$ Concentração do fluopicolida na solução em equilíbrio no processo de dessorção $\left(\mathrm{mg} \mathrm{L}^{-1}\right)$; $V=$ Volume de solução de $\mathrm{CaCl}_{2} 0,01 \mathrm{M}$, sem fluopicolida adicionada ao solo para a dessorção $(\mathrm{mL})$.

\section{RESULTADOS E DISCUSSÃO}

\subsection{MINERALIZAÇÃO DO FLUOPICOLIDA}

A Figura 1 apresenta a produção de ${ }^{14} \mathrm{CO}_{2}$ resultante da ação microbiana sobre 0 ${ }^{14} \mathrm{C}$-fluopicolida durante o período de incubação de até 130 dias após a aplicação do fungicida ao solo.

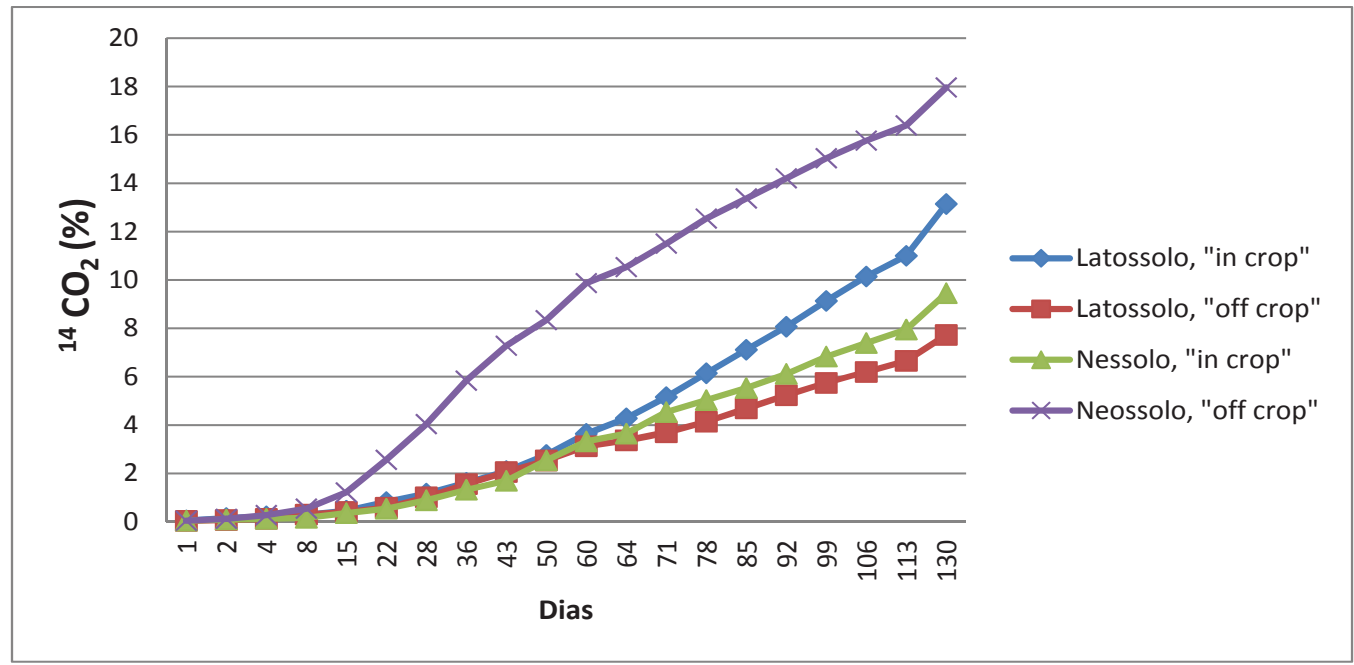

FIGURA 1 - MINERALIZAÇÃO DO FLUOPICOLIDA

("in-crop"= área tratada; "off-crop" = área preservada).

As Tabelas 4 e 5 apresentam os resultados da mineralização, extração e combustão das amostras.

\section{TABELA 4 - RECUPERAÇÃO APÓS APLICAÇÃO DE ${ }^{14}$ C-FLUOPICOLIDA EM LATOSSOLO}

\begin{tabular}{|c|c|c|c|c|c|}
\hline $\begin{array}{l}\text { Tempo de } \\
\text { Incubação } \\
\text { (dias) }\end{array}$ & Amostras & $\begin{array}{c}{ }^{14} \mathrm{C} \text {-Extraíveis } \\
(\%)\end{array}$ & ${ }^{14} \mathrm{C}$-Ligados (\%) & $\begin{array}{l}{ }^{14} \mathrm{CO}_{2} \\
(\%)\end{array}$ & $\begin{array}{c}{ }^{14} \mathrm{C} \text { - Total } \\
(\%)\end{array}$ \\
\hline \multirow{2}{*}{0} & "in crop" & 102 & 2 & - & $104 \pm 4,9$ \\
\hline & "off crop" & 99 & 9 & - & $108 \pm 5,4$ \\
\hline \multirow{2}{*}{30} & "in crop" & 98 & 3 & 1,2 & $102 \pm 1,6$ \\
\hline & "off crop" & 100 & 6 & 1,0 & $107 \pm 8,4$ \\
\hline \multirow{2}{*}{60} & "in crop" & 91 & 6 & 3,6 & $101 \pm 1,1$ \\
\hline & "off crop" & 95 & 8 & 3,1 & $106 \pm 1,6$ \\
\hline \multirow{2}{*}{130} & "in crop" & 81 & 6 & 13,1 & $100 \pm 8,6$ \\
\hline & "off crop" & 84 & 11 & 7,7 & $103 \pm 10,4$ \\
\hline
\end{tabular}

("in-crop"= área tratada; "off-crop" = área preservada). 
TABELA 5 - RECUPERAÇÃO APÓS APLICAÇÃO DE ${ }^{14}$ C-FLUOPICOLIDA EM NEOSSOLO

\begin{tabular}{|c|c|c|c|c|c|}
\hline $\begin{array}{l}\text { Tempo de } \\
\text { Incubação } \\
\text { (dias) }\end{array}$ & Amostras & $\begin{array}{c}{ }^{14} \mathrm{C} \text {-Extraíveis } \\
(\%)\end{array}$ & ${ }^{14} \mathrm{C}$-Ligados (\%) & $\begin{array}{l}{ }^{14} \mathrm{CO}_{2} \\
(\%)\end{array}$ & $\begin{array}{c}{ }^{14} \mathrm{C} \text { - Total } \\
(\%)\end{array}$ \\
\hline \multirow{2}{*}{0} & “in crop" & 103 & 1 & - & $104 \pm 1,2$ \\
\hline & "off crop" & 105 & 1 & - & $106 \pm 0,9$ \\
\hline \multirow{2}{*}{30} & “in crop” & 91 & 2 & 0,9 & $94 \pm 7,4$ \\
\hline & "off crop" & 111 & 2 & 4,0 & $117 \pm 11,9$ \\
\hline \multirow{2}{*}{60} & “in crop” & 87 & 3 & 3,3 & $93 \pm 14$ \\
\hline & "off crop" & 82 & 4 & 9,9 & $96 \pm 6,9$ \\
\hline \multirow{2}{*}{130} & “in crop" & 88 & 4 & 9,5 & $101 \pm 4,6$ \\
\hline & "off crop" & 77 & 5 & 18,0 & $100 \pm 4,3$ \\
\hline
\end{tabular}

("in-crop"= área tratada; "off-crop" = área preservada).

Para o solo Latossolo, nas áreas "in crop" e "off-crop", não ocorreu diferença significativa entre os resultados da mineralização até 130 dias de experimento $(p>0,05, p=0,348)$. Em relação ao Neossolo, observou-se diferença significativa entre os resultados obtidos para as áreas "in-crop" e "off-crop" durante todo o período de incubação e isso foi confirmado pela análise de variância ANOVA $(p<0,05, p=0,022)$ (Tabela 6$)$.

Conforme Oliveira \& Brighenti (2011), o pH pode influenciar o comportamento dos solos e a atividade microbiológica é regulada pelas condições de solo, particularmente seu conteúdo de água, temperatura e pH. A mudança de pH da área "in-crop" devido à fertilização em Neossolo pode ter alterado a atividade microbiana e por isso a taxa de mineralização foi menor na área "in-crop" quando comparada à área "off-crop. Deve-se lembrar que as características físico-químicas dos solos e as da própria molécula influenciam o comportamento dos agrotóxicos e sua dissipação no solo (LUCHINI \& ANDRÉA, 2002).

Os dois solos, independentemente das áreas, produziram mineralização do composto inferior a $20 \%$ no período de 130 dias. Somente a taxa de mineralização não indica persistência do fluopicolida no solo, pois sua degradação pode ser mais rápida que a taxa de mineralização como, por exemplo, pela formação de metabólitos.

Quando se comparam os resultados obtidos por mineralização com os dados disponíveis no relatório emitido pelo European Food Safety Authority (EFSA, 2009) para o mesmo composto, verifica-se maior mineralização e menor formação de resíduos ligados nos solos tropicais utilizados neste trabalho, indicando maior degradação do agrotóxico em solos tropicais. No mesmo relatório (EFSA, 2009), há resultados de trabalhos realizados para investigar o comportamento de dissipação do fluopicolida em condições de campo que estimaram a meia vida $\left(t_{1 / 2}\right)$ do produto entre 50 e 172 dias. A obtenção de meia vida menor em condições laboratoriais indica que o fluopicolida, provavelmente, terá dissipação mais rápida no ambiente.

\subsection{SORÇÃO E DESSORÇÃO DO FLUOPICOLIDA}

A Tabela 6 apresenta os resultados obtidos nos ensaios de sorção e dessorção do fluopicolida nos solos Latossolo e Neossolo, respectivamente. 
TABELA 6 - PARÂMETROS AVALIADOS NOS ENSAIOS DE SORÇÃO E DESSORÇÃO DO FLUOPICOLIDA NO LATOSSOLO E NEOSSOLO

\begin{tabular}{cccccc}
\hline Solo & \multicolumn{2}{c}{ Coeficientes de Freundlich } & & $\mathbf{K}_{\mathrm{foc}}$ & $\mathbf{A}_{\mathrm{D}}$ \\
\hline $\begin{array}{c}\text { Latossolo "off } \\
\text { crop" }\end{array}$ & $12, \mathbf{K}$ & 0,9828 & 0,9944 & 273,20 & 0,040 \\
\hline $\begin{array}{c}\text { Latossolo "in } \\
\text { crop" }\end{array}$ & 10,03 & 0,9588 & 0,996 & 223,91 & 0,036 \\
\hline $\begin{array}{c}\text { Neossolo "off } \\
\text { crop" }\end{array}$ & 4,48 & 0,9093 & 0,995 & 354,58 & 0,042 \\
\hline $\begin{array}{c}\text { Neossolo "in } \\
\text { crop" }\end{array}$ & 3,16 & 0,8952 & 0,986 & 237,27 & 0,038 \\
\hline
\end{tabular}

Nos resultados obtidos para o coeficiente normalizado de Freundlich para a adsorção $\left(\mathrm{K}_{\mathrm{f}}\right)$, em relação ao carbono orgânico para ambas as áreas dos solos Neossolo e Latossolo, os valores de $\mathrm{K}^{\text {foc }}$ situaram-se entre 223,9 e $354,6 \mathrm{~mL} \mathrm{~g}^{-1}$. O coeficiente de determinação $\left(R^{2}\right)$ mostrou que a isoterma da adsorção é adequada para o modelo de Freundlich.

Pela classificação do EFSA (2009), o fluopicolida apresentou média mobilidade de acordo com o coeficiente de adsorção normalizado $\mathrm{K}_{\text {foc }}$. $\mathrm{O}$ fungicida mostrou comportamento similar nos solos brasileiros Latossolo e Neossolo quando comparado aos reportados na avaliação daquela instituição (faixa de $\mathrm{K}_{\text {foc }}$ de $172-580 \mathrm{~mL} . \mathrm{g}^{-1} \mathrm{em}$ nove solos). Com base no resultado da constante de adsorção obtido, a classificação do fluopicolida de acordo com o IBAMA (1996), seria a de molécula com média adsorção para o Latossolo (ambas as áreas) e baixa adsorção para o Neossolo (ambas as áreas).

\section{CONCLUSÃo}

A dissipação do fluopicolida nos solos estudados foi menor que $20 \%$ até 130 dias, indicando que o fungicida não é rapidamente mineralizado pelos micro-organismos nos solos estudados. Não foi verificada diferença significativa para a mineralização do fluopicolida no solo Latossolo em áreas "in crop" e "off crop". Já para o Neossolo, foi observada diferença significativa na mineralização do fungicida entre suas áreas agrícolas e preservada, tendo provavelmente o $\mathrm{pH}$ influenciado a atividade microbiana.

Em relação aos valores de adsorção obtidos, pode-se concluir que a molécula do fungicida fluopicolida apresenta média adsorção para o solo Latossolo e baixa adsorção para o Neossolo.

Os resultados de mineralização do ${ }^{14} \mathrm{C}$-fluopicolida, resíduos extraíveis, resíduos ligados, aplicações repetidas e a adsorção do composto evidenciaram que não houve diferença no comportamento do fungicida em relação às áreas "in-crop" e "off-crop" nos solos estudados. Concluiu-se que o fluopicolida não exerce efeito adverso em áreas nas quais há constante manejo agrícola em razão de seu comportamento similar em área preservada.

\section{ABSTRACT \\ MINERALIZATION AND SORPTION OF FLUOPICOLIDE FUNGICIDE ON SOILS SAMPLES OF NEOSSOLO AND LATOSSOLO FROM OFF-CROP AND IN-CROP AREAS}

The objective of this study was to verify the mineralization and sorption of flupicolide fungicide on Neossolo and Latossolo from off-crop and in-crop areas in order to compare and identify alterations on pesticide behavior in different soils and areas. For all soil samples, mineralization rates were $<20 \%$, although for Neossolo there 
was a higher mineralization on off-crop area when compared to the respectively in-crop area. This behavior was not observed for Latossolo because both areas had similar mineralization behavior. The results of adsorption coefficients presented a medium and a low adsorption for Latossolo and Neossolo respectively in both areas. In addition, the sorption isotherms of fluopicolide were well adjusted to Freundlich model, with 1/n around 0.9. Based on these results, fluopicolide can be considered a fungicide with slow mineralization and medium mobility for Neossolo and Latossolo and on in-crop and off-crop areas. It was concluded that there is no adverse effect of the use of fluopicolide in agricultural management areas.

KEY-WORDS: PESTICIDES; MINERALIZATION; SORPTION; SOILS; FLUOPICOLIDE.

\section{REFERÊNCIAS}

1 COX, L.; HERMOSIN, M.C.; CELIS, R.; CORNEJO, J. Sorption of two polar herbicides in soils and soil clays suspensions. Water Research, New York, v.31, p.1309-1316, 1997.

2 GREEN, R.E.; KARICKHOFF, S.W. Sorption estimates for modeling. In: CHENG, H.H. Pesticides in the soil environment: processes, impacts, and modeling. Madison: Soil Science Society of America, 1990. p. 79-101.

3 European Food Safety Authority (EFSA). Peer review of the pesticides risk assessment of the active substance fluopicolide. Parma, 2009. p. 1-58. (EFSA Scientific Report, 299).

4 FRIGHETTO, R.T.S.; VALARINI, P.J. Indicadores biológicos e bioquímicos da qualidade do solo. In: MANUAL técnico da Embrapa. Jaguariúna, 2000. p. 37-40.

5 Instituto Brasileiro do Meio Ambiente e dos Recursos Naturais Renováveis (IBAMA). Manual de testes para avaliação da ecotoxicidade de agentes químicos. Brasília, 1990, 351 p. Part E3. (Teste de avaliação da adsorção/dessorção, revisão1996)

6 LAVORENTI, A. Comportamento de herbicidas no meio ambiente. In: WORKSHOP DE BIODEGRADAÇÃO, Campinas, 14-16 de outubro de 1996. Anais.... Campinas: Embrapa, 1996.

7 LUCHINI, L. C; ANDRÉA, M. M. Dinâmica de agrotóxicos no ambiente. In: FÓRUM NACIONAL DE SECRETÁRIOS DE MEIO AMBIENTE. Textos Orientadores... Brasília: Ministério da Agricultura, 2002 p. 27-44.

8 MICROSOFT CORPORATION. Microsoft office - Exel 2010. S.I., 2010.

9 MESQUITA, T.B; RÜEGG, E.F. Influência de agentes tensoativos na detecção da radiação beta. Ciênc. e Cult., São Paulo, v.36, p.446-450, 1984.

10 NAKAGAWA, L.E; ANDRÉA M.M. Liberação de resíduos não extraíveis ou ligados do herbicida atrazina em solo e sua absorção por plantas. Pesquisa Agropecuária Brasileira, Brasília, v.35, n.8, p.1517-1522, ago. 2000.

11 Organisation for Economic and Co-operation and Development (OECD). Guideline for testing of chemicals: part. 304A. S.I., 1981.

12 Organisation for Economic and Co-operation and Development (OECD). Guideline for testing of chemicals: part. 106. S.I., 2000.

13 OLIVEIRA, M.F.; BRIGHENTI, A.M. Comportamento dos herbicidas no ambiente. Curitiba, PR: Omnipax, 2011. p.263304.

14 SPADOTTO, C.A; GOMES, M.A.F; LUCHINI, L.C.; ANDRÉA, M.M. Monitoramento do risco ambiental de agrotóxicos: princípios e recomendações. Jaguariúna: EMBRAPA, 2004. 29 p. 